\title{
On Polymers Nanocomposites: Electrical Treeing, Breakdown models and Related Simulations
}

\author{
G. Melissinos \\ Democritus University of Thrace \\ Department of Electrical and Computer Engineering \\ Power Systems Laboratory, Xanthi, Greece \\ melissinosgeorg@gmail.com
}

\author{
M. G. Danikas \\ Democritus University of Thrace \\ Department of Electrical and Computer Engineering \\ Power Systems Laboratory, Xanthi, Greece \\ mdanikas@ee.duth.gr
}

\begin{abstract}
This paper deals with polymer nanocomposites and their related breakdown mechanisms. Polymer nanocomposites seem to be a very promising alternative to conventional polymers regarding high voltage applications. Some developed breakdown models are discussed as well as the mechanism of treeing in such materials. Treeing simulation results are presented.
\end{abstract}

Keywords-polymer; nanocomposites; insulation; treeeing; breakdown.

\section{INTRODUCTION}

Polymeric materials have been used for a long time in high voltage engineering [1]. Polymer nanocomposites were proposed more than twenty years ago as a plausible alternative to traditional polymeric materials [2] but their full potential was realized more recently [3]. It was realized that the unique properties of polymer nanocomposites were due to the interfaces formed between the nanoparticles and the surrounding polymer matrix. For this reason, it was emphasized that it was mandatory that the coupling of the interfacial region and the base polymer was crucial for the development of new insulating materials [4-6]. In polymer nanocomposites the nanoparticles are homogenously dispersed in the matrix in concentrations $<10 \mathrm{wt} \%$, the size of particles being smaller than $100 \mathrm{~nm}$.

The interfacial region in the nanocomposites is significantly increased in comparison to microcomposites, due to the nanometric scale of the particles, which means that the augmentation of the interfacial region gives to nanocomposites different and unique properties compared to microcomposites. Nanoparticles can improve or adjust significantly different properties, such as electrical, mechanical, thermal, and optical but with the condition to be homogenously dispersed in the polymer matrix. A very good dispersion of the nanoparticles into the polymer matrix can be achieved by using different methods and preparation techniques among them mechanical dispersion methods, including ultrasonic vibration or special sol-gel techniques, high shear energy dispersion mixing and/or through a tailored surface modification of the nanoparticles [7]. Lack of compatibility between the polymer matrix and the nanoparticles may cause the formation of agglomerations [8].
For applications in high voltage engineering, it is imperative that such polymer nanocomposite materials have high breakdown strength and a high resistance to electrical treeing. It is the purpose of this paper to review some of the published breakdown and treeing models as well as to give some simulation results regarding treeing in nanocomposites.

\section{BREAKDOWN AND DEGRADATION IN POLYMER NANOCOMPOSITES}

At breakdown, the electric field frees bound electrons. If the applied electric field is sufficiently high, free electrons from background radiation may become accelerated to velocities that can liberate additional electrons during collisions with neutral atoms or molecules in a process called avalanche breakdown. Breakdown occurs quite abruptly, resulting in the formation of an electrically conductive path, thus destroying the insulating capability of the material. The first approach, an effort made so that the breakdown strength be explained on quantum mechanics level, was proposed in [9] that suggested the low energy criterion, which says that fast avalanche breakdown can only occur if the average energy gained of electrons are larger than the average energy losses of electrons. Von Hippel' $s$ theory was the basis for the density functional perturbation theory (DFPT), for estimating the breakdown of new insulating materials [10] and also for the determination of the depths of impurity states created by common chemical impurities [11]. Depths of traps/hopping sites were between $0.1 \mathrm{eV}$ and $2.4 \mathrm{eV}$ and were classified into shallow traps/hopping sites, deep traps, and deeper traps. Such depths suggest that chemical impurities can play major roles in trapping and hopping processes, can explain the observed activation energy of conduction, and shape the barriers to charge injection. The type of impurity bonds and their lengths are correlated with the depth of impurities they create. Such correlation is linear [11].

In 2005, Ma and others showed in experiments with LDPE/TiO2 nanocomposite, that the breakdown strength improved after the treatment of nanoparticles surface with silanes, which promoted deep trapping of charges [12]. Han and others, in their experiments with LDPE/NaY nanocomposite with $3 \mathrm{wt} \%$ zeolite nanoparticles, concluded that deep traps that create homo space charges can suppress the injection of charges from the electrodes, decrease the mobility 
of charge carriers resulting in improved breakdown strength [13]. Classical theories of breakdown may apply to polymer nanocomposites, such as the electrical avalanche breakdown [14], the water treeing breakdown theory [15], the electrical treeing breakdown theory [16], the thermal breakdown theory [17] and the electromechanical breakdown theory [18]. It happens, however, that in certain cases, the above mentioned models can be intermingled, as is the case of [19], where it was pointed out that, for PE and EVA polymer samples, thermal breakdown happens along with dominating fast avalanche mechanism at low temperatures and a dominating electromechanical mechanism at higher temperatures, depending on the temperature level.

It is to be remembered that besides such classical models of breakdown, in the case of polymer nanocomposites, a crucial role plays the interface between the polymer matrix and the nanoparticles. The improved nanodielectric properties seem to stem from the immensely enlarged interface region around the nanoparticles, in usually low concentrations (1-10 wt \%) in comparison of the interface regions of microcomposites with the same concentrations. In a simple example, Kickelbick [20] demonstrated the importance of the interface in nanoscale level, as almost every nanoparticle in the nanocomposite, has surface that interacts with the polymer matrix. Generally speaking, nanocomposites seem to have higher breakdown strength than their conventional counterparts. In the following chapter, interface models and related problems and interpretations will be studied.

\section{INTERFACES IN NANOCOMPOSITES}

Apparently the degrading mechanisms of the polymers, such as partial discharges (PD) and electrical and/or water treeing, can also lead polymer nanocomposites to destructive failure [21]. In [22], authors suggested that the advantageous nanocomposite properties have their origins in the behavior of the interfacial interaction zone surrounding the nanoparticles. In this regard, two main features would appear to be dominant a) the mobility ascribed to the physical and chemical bonding occurring at the interface, and b) the formation of a double layer in the interfacial region which can influence local conductivity. The mobility and the type and degree of crystallinity changes with nanoparticle addition in the polymers [23]. Moreover the polymeric chain mobility in the interface zone is affected by many factors, such as the polymeric chain length [21], the morphology of the nanoparticle surface and the polymer-particle interactions (repulsive or attractive) [24], the kind of polymer-particle chemical bonds [25] and the wt $\%$ nanoparticle concentrations [26, 27]. In [28], authros observed in nanocomposite with silica nanoparticles, two glass transition temperatures (GTT), and they proposed for the explanation of these morphological and structural anomalies a model according to which the lower GTT was attributed to the usual polymeric regions, while the higher to the interface zone with the reduced mobility. For concentrations of nanoparticles more than $10 \mathrm{wt} \%$ the mobility is considerably decreased, while for above $20 \mathrm{wt} \%$ the polymeric chains are almost immobilized. In [29], authors suggested that in order for the voltage endurance to be increased in a nanocomposite, internal charges should be decreased through interfacial effects and if the interaction zone is the primary agency in determining the electrical behavior, then clearly the engineering of nanodielectrics should he centered on chemical modifications of the interface.

In [12], the authors presented the results of the influence of surface modification of $\mathrm{TiO}_{2}$ nanoparticles on the shortbreakdown strength and space charge distribution of LDPE. They concluded that the improved dielectric breakdown strength was directly due to beneficial effects related to the variation of the electrical features at the particle surface due to introduction of polar groups. For the same reason, with the use of surface modified nanoparticles, the formation of space charge was suppressed. In [30], it was reported that the deep traps for electron carriers which are formed by additives in polymers, reduced the energy of space charges, while the created homocharges hindered further charge injection from the electrodes, which improved the breakdown strength. In a later work [31], the authors presented a hypothesis for the mechanisms that lead to the improved AC, DC and impulse breakdown strength of composite XLPE with nanoparticles $\mathrm{SiO}_{2}$. They suggested that 'the inclusion of nanoparticles provides myriad scattering obstacles and trap sites in the charge carriers' paths, effectively reducing carrier mobility and thus carrier energy. The result is homocharge buildup at the electrodes, which increases the voltage required for further charge injection due to blocking by the homocharge'. Scattering, caused by both particle/polymer interfaces and possibly disrupted crystalline structure, contributes to the better electrical behavior of nanocomposites besides the defect reduction via chemical linkage between nanoparticles and polymer or the introduction of deep trap sites in the polymer due to the surface modifier groups [32].

From all the above reports it is clear that there are many factors that influence the breakdown strength of nanocomposites, but the involved dominant breakdown mechanism is partly dependent on the temperature conditions, as for example a temperature increase will reduce the polymer mechanical strength. Regarding water tree retardation in nanocomposites, the addition of $\mathrm{MgO}$ nanoparticles in LDPE and XLPE in different concentrations managed to improve the nanocomposite strength in water tree growth as well as to observe the temperature effect on its development [33]. In [34, 35] the influence of the interface mechanical strength in barium titanate-polyvinydene fluoride nanocomposite breakdown strength was investigated. In the interface area the titanate covered bariun nanoparticles were cross linked to the polyvinydene fluoride matrix forming an organically integrated body, which posed a potential barrier which prevented charge carriers to cross through the interface area. The charge carriers are confined in the polymer area only, which promotes energy storage, while, with the tetrafluorophthalic acid nanoparticle treatment, a passivation layer around the interfacial area is formed, thus reducing the generation of charge carriers which may results in ionic or dc conduction and the decrease of the breakdown strength. Yet in other publications, it was proved that nanoparticle treatment which resulted in covalently-bonded interface polymer-particle nanocomposites exhibit improved breakdown strength $[36,37]$. It is quite clear from the above 
that interfaces and their treatment are dominant factors in defining the breakdown strength of polymer nanocomposites.

Interface models were proposed in the past that tried -at least partially - to interpret the behavior of polymer nanocomposites. This subject was already partly treated [38, 39] and it will be treated in more detail separately in a future publication, consequently interface models only briefly will be mentioned here. Interface models are extremely useful for better understanding the interfacial properties of nanocomposites and they basically treat the areas around the nanoparticles, offering thus interpretations as to why polymer nanocomposites have improved electrical behavior compared to their conventional counterparts. Main interface models referring to nanocomposites - are the Lewis model, the Wilkes model, the Tanaka multi-core model, Tsagaropoulos' model, the potential barrier model, the interphase model, the polymer chain alignment model, the water shell model, the dual layer model, the physical model for the explanation of surface degradation mechanism, the physical model for tree growth, Chen's model and the percolation model. All the above mentioned models - as said to be discussed in a future paperrepresent a significant effort towards the understanding of the properties that emerge in polymer nanocomposites. There is not, however, a universal model so far, that will cover all the experimental facets that are simulated by all these models.

\section{BREAKDOWN AND RELATED MECHANISMS}

Various models have been proposed for the breakdown of polymers. Partial discharges (PD) is a major source of electrical degradation and occur in voids, de-laminations and other defects in an insulation system. PD may cause electrical treeing. Electrical treeing is the main indication of aging and ultimately leads to insulation breakdown and eventual failure of the electrical equipment. Various models have been proposed for the breakdown of polymers. Niemeyer, Pietronero and Wiesmann (NPW) [40, 41] introduced a stochastic model in which the evolution of the tree-like patterns are explicitly depend on local electric field strength and a factor which modulates the randomization of the process. The fractal dimensions of the experimentally obtained tree-like patterns, are in general, in accordance with the patterns obtained from the breakdown simulations, which advocates in favor of the fractal characteristic of the electrical trees. An improved model was proposed in [42], where there was a generalization by introducing a breakdown criterion for tree formation, so that the branches could only be added if the local field at a branch tip exceeded a critical threshold field for growth, and by introducing an internal field within the tree channels that would add to the electrode potential at the tip of the tree, implying thus that the tree channel could not be considered without resistance. More recently, Dodd [43] presented his deterministic model which s successful in simulating electric trees in dielectric without the need of a random variable, a requirement of previous stochastic models. Instability in the spatial development of partial discharges within the tree structure takes the role of the stochastic element as used in previous models to produce branched tree structures. In yet another model, electric treeing is simulated with the use of von Neumann Cellular Automata (CA) theory. More specifically, it is presumed that electric charges in the electrode tip create electric field that surpasses the dielectric strength of the polymer insulator and a gradual tree-like channel formation is spread in it. On every step the local potential on every point is being recalculated, due to local dielectric permittivity fluctuations caused by the tree channel propagations [44, 45]. Dissado and Sweeney [46] proposed a deterministic quantitative physical model for the breakdown of solids by discharges originating from voids or channel tips. It is found that fractal tree-like structures are formed only when the local electric fields at the tree tips are allowed to fluctuate about their Laplace values. By considering the local-field enhancement factor as a white noise, which is produced by the breakdown mechanism itself, it is shown that the amount of branching depends only on the range of fluctuations allowed.

Noskov' s model took into account PD erosion or the real distribution of the electric field due to space charges, and also that the electric trees had to emanate always from matrix defects . The model could estimate electrical tree propagation and PD activity within growing tree channels. The local electric field and the damage accumulation in the dielectric material were constituent into the tree growth, while the increase of the damage was proportional to the PD energy release in the channels. The electric field distribution was determined by the charge deposition within the tree structure and the electrode geometry, while charge distribution changes within the channels during PD. PD starts when the electric field along the channels exceeds a threshold inception value and stops when the field falls below the threshold quenching value [47]. Although the above mentioned publications refer mainly to conventional polymers, their ideas could also apply to nanocomposites. The CA model, for example, was applied to simulate electrical treeing in nanocomposites [48]. However, a drawback of the CA model is that it does not consider in detail the interface region around the nanoparticles. On the other hand, this is understandable since there is no generally accepted model for the interfaces as yet. Generally speaking, in the various models, in order an electrical tree simulation to take place in nanocomposites, local fluctuations are used, flactuations of factors that simultaneously comprise variables of functions that determine the conditional representation of the discrete cells of the polymer matrix and of the nanoparticles of the nanocomposite. Factors that can be used as variables, are the electric field or the released energy due to PD activity, with the first to be greatly preferred because it can be easily calculated. The CA model considers as decisive factor the local change -even slightly- of the dielectric constant that in turn affects the local electric field [48].

\section{TREEING SIMULATIONS}

Treeing simulations in nanocomposites were based on the model developed in [48]. As nanocomposites consist of different materials, it is only logical to try to develop the simulations on previous treeing models of conventional polymers [44, 45]. More specifically, the initial CA model for nanodielectrics, had analysis 5000x5000 cells, a point-plane electrode arrangement and every nanoparticle was presented in just one cell. Generally the design concepts of a previous model $[44,45]$ are applied to nanocomposite treeing simulation 
model, with in addition, the presence of some nanoparticles around the tip of high voltage electrode. Cell's condition is given by $C_{i, j}=\left\{E F_{i, j}, T F_{i, j}, g_{i, j}, V_{i, j}\right\}$ where $E F_{i, j}$ denotes whether the cell belongs to the nanocomposite or electrode, $T F_{i, j}$ denotes tree cells, $g_{i, j}$ cell's inhomogeneity factor and $V_{i, j}$ cell's potential. The electric field is calculated by $E=g \Delta V / d$, where $\mathrm{g}$ the inhomogeneity factor taking random values between 0,9 $1,1, \mathrm{~d}$ the distance between neighbor cells and $\Delta V$ the potential between them.

For electric tree propagation an algorithm is executed first to the cells at the electrode tip and when the electric field exceeds the nanocomposite breakdown strength, the cells are converted to tree cells and this procedure is repeated to all cells until the tree propagation stops. The cell potential is calculated solving Laplace or Poisson equations as well as the boundary conditions determined as described in [44, 45]. The potential distribution is also affected by interactions between the nanoparticles due to their proximity. Cox in his work [49] in a model with two spheres calculated accurately enough sphere potentials $\Phi_{A}, \Phi_{B}$, with $E$ the external electric field, $d$ the distance between sphere centers, $a$ sphere radius, $k$ the sphere permittivity ratio to that of the polymer, $\lambda=\cos \theta, \Lambda=\cos \Theta$ and $P_{n} a$ Legendre $\mathrm{n}$ grade polyonym. When in the nanocomposite there is a defect or microparticle the potential is $\Phi=-\left(3 /\left(\left(\varepsilon_{2} / \varepsilon_{1}\right)\right.\right.$ +2)) $E r \cos \theta$, where $\varepsilon_{1}$ the polymer permittivity and $\varepsilon_{2}$ that of the microparticle while $r$ is it's radius and $\theta$ the angle between the electric field vector and radius.

$$
\begin{gathered}
\Phi_{A}=\frac{E_{d}}{2}-\sum_{n=1}^{\infty} a_{n}\left[\left(\frac{a}{d}\right)^{n+1}+\frac{2 n+1}{n(k-1)}(1)^{n} P_{n}(\lambda)\right] \\
\Phi_{B}=-\frac{E_{d}}{2}-\sum_{n=1}^{\infty} a_{n}\left[\left(\frac{a}{d}\right)^{n+1}+\frac{2 n+1}{n(k-1)}(-1)^{n} P_{n}(\Lambda)\right]
\end{gathered}
$$

Simulations -regarding the influence of space charges on the development of electrical treeing- with the model described briefly above as well as in [48] gave results qualitatively not different from those reported in [50, 51]. Specifically, it was shown that space homocharges around the high voltage tip electrode, hinder further charge diffusion from the electrode into the matrix, as well as electric tree growth and breakdown of the nanodielectric. It was shown also that electrical tree deployment through the nanocomposite, was taking place only through the polymer matrix deviating from the nanoparticles, which increased significantly the lengths of electrical tree paths to the opposite electrode, resulting in increased breakdown strength and delayed breakdown. Furthermore, the effect of the size of nanoparticles included in a nanocomposite was simulated (Figures 1 and 2). It is evident that smaller size nanoparticles (which means more nanoparticles per volume) have a beneficial effect on hindering electrical treeing, in accordance with experimental results published in [52]. In other words, an increase in wt\% nanoparticle content, offers a longer treeing path and thus a higher breakdown strength. It must, however, kept in mind that not only the size of nanoparticles in important but also, among other factors, the coupling agents. Coupling agents in nanocomposites are more effective in small sizes of nanoparticles and increase tree breakdown time [52]. An interesting parameter which was investigated was the presence of air voids in a nanocomposite (Figures 3 and 4).

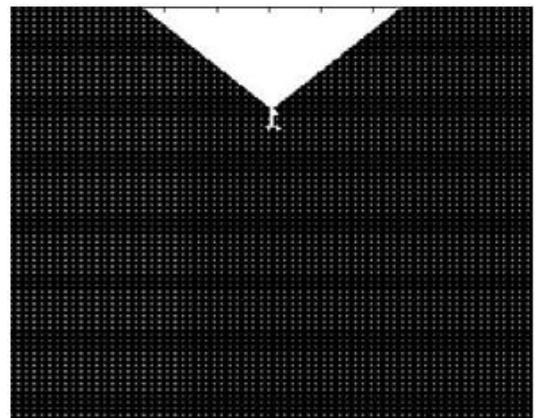

Fig. 1. Electrical tree propagation in epoxy/TiO2 nanocomposite with 100 nm nanoparticles diameter

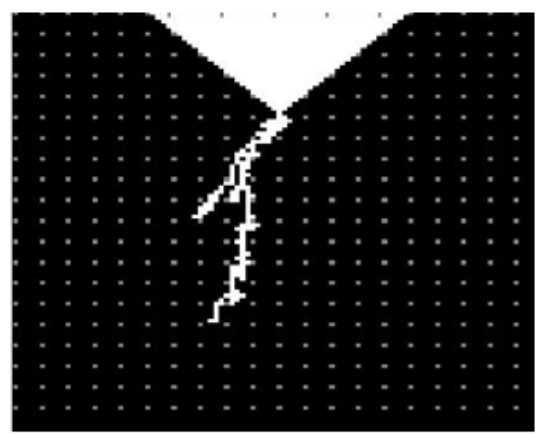

Fig. 2. Electrical tree propagation in epoxy/TiO2 nanocomposite with $200 \mathrm{~nm}$ nanoparticles diameter

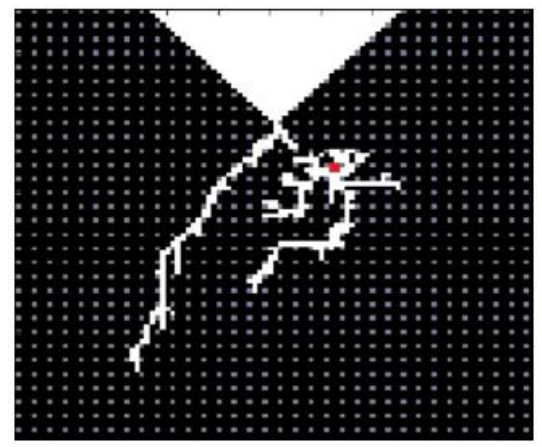

Fig. 3. Tree propagation in a nanocomposite (epoxy resin with $\mathrm{TiO} 2$ nanoparticles) in the presence of one air-filled void

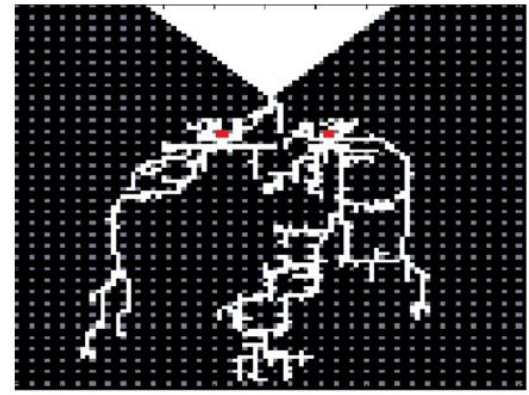

Fig. 4. Tree propagation in a nanocomposite (epoxy resin with $\mathrm{TiO} 2$ nanoparticles) in the presence of two air-filled voids 
Simulations with the model described above [48], indicated that voids cause -as in conventional polymers- problems. As shown in Figures 3 and 4, in the presence of an air void, electrical treeing is encouraged despite the existing nanoparticles. With two air-filled voids, treeing propagation becomes even worse. The outcome of all the above simulations and cited technical literature converge to a general conclusion, that the increase of the nanoparticle content -up to a certain percentage- may impede electric tree propagation and $\mathrm{PD}$ activity and erosion in nanocomposites. However, even nanocomposites must avoid defects, such as air-filled voids, that are detrimental to all kinds of insulation systems.

\section{CONCLUSIONS}

In this paper, a background as to the some aspects of polymer nanocomposites was given. Some advantages of these materials as well as some of their problem areas were commented upon. Simulations of electrical tree propagation, based on a CA model, were presented. It was shown that the size of the nanoparticles plays a crucial role in determining the treeing path and propagation. It was also pointed out that conventional polymers as well as polymer nanocomposites "suffer" both from defects, such as air-filled voids. Experimental results seem to justify some of the simulations shown here.

\section{REFERENCES}

[1] G. C. Stone, E. A. Boulter, I. Culbert, H. Dhirani, "Historical development of insulation materials and systems", in: Electrical insulation for Rotating Machines Design, Evaluation, Aging, Testing, and Repair, 1st Edition, Editor: S. V. Kartalopoulos, Wiley-IEEE Press: Piscataway, NJ, USA, pp. 73-94, 2004

[2] T. J. Lewis, "Nanometric Dielectrics", IEEE Transactions on Dielectrics and Electrical Insulation, Vol. 1, pp. 812-825, 1994

[3] J. K. Nelson, J. C. Fothergill, "Internal charge behaviour in nanocomposites", Nanotechnology, Vol. 15, pp. 586-595, 2004

[4] T. Tanaka, G. C. Montanari, R. Muelhaupt, "Polymer nanocomposites as dielectrics and electrical insulation: Perspectives for processing technologies, material characterization and future applications", IEEE Transactions on Dielectrics and Electrical Insulation, Vol. 11, pp. 763784, 2004

[5] Y. Cao, P. C. Irwin, K. Younsi, "The future of nanodielectrics in the electrical power industry", IEEE Transactions on Dielectrics and Electrical Insulation, Vol. 11, pp. 797-807, 2004

[6] M. F. Frechette, M. L. Trudeau, H. D. Alamdar, S. Boily, "Introductory remarks on nanodielectrics", IEEE Transactions on Dielectrics and Electrical Insulation, Vol. 11, pp. 808-818, 2004

[7] I. Plesa, P. V. Notingher, S. Schlögl, Ch. Sumereder, M. Muhr, "Properties of polymer composites used in high-voltage applications", Polymers, Vol. 8, pp. 1-63, 2016

[8] R. Y. Hong, Q. Chen, "Dispersion of inorganic nanoparticles in polymer matrices: challenges and solutions", in: Organic-Inorganic Hybrid Nanomaterials, Eds. S. Kalia and Y. Haldorai, Springer International Publishing: Berlin, Heidelberg, Germany, pp. 1-38, 2015

[9] A. von Hippel, "Electric breakdown of solid and liquid insulators", Journal of Applied Physics, Vol. 8, pp. 815-832, 1937

[10] Y. Sun, S. A. Boggs, R. Ramprasad, "The intrinsic electrical breakdown strength of insulators from first principles", Applied Physics Letters, Vol. 101, pp. 1329061-1329065, 2012

[11] A. Huzayyin, S. Boggs, R. Ramprasad, "Depths of chemical impurity states in polyethylene the big picture from first principles", in : IEEE
International Conference on Solid Dielectrics, Bologna, Italy, Vol. 1, pp. 15-18, 30th June - 4th July, 2013

[12] D. Ma, T. A. Huneger, R. W. Siegel, A. Christerson, E. Martensson, C. Onneby, L. S. Schadler, "Influence of nanoparticle surface modification on the electrical behavior of polyethylene nanocomposites", Nanotechnology, Vol. 16, pp. 724-731, 2005

[13] B. Han, X. Wang, Z. Sun, J. Yang, Q. Lei, "Space charge suppression induced by deep traps in polyethylene/zeolite nanocomposite", Applied Physics Letters, Vol. 102, pp. 012902-1 - 012902-4, 2013

[14] Y. Tanaka, N. Ohnuma, K. Katsunami, Y. Ohki, "Effects of crystallinity and electron mean-free-path on dielectric strength of low-density polyethylene", IEEE Transactions on Electrical Insulation, Vol. 26, No. 2, pp. 258-265, 1991

[15] Z. H. Fan, N. Yoshimura, "The influence of crystalline morphology on the growth of water trees in PE", IEEE Transactions on Dielectrics and Electrical Insulation, Vol. 5, No. 3, pp. 849-858, 1996

[16] T. Tanaka, 'Interface properties and surface erosion resistance', pp. 229258, in: Dielectric Polymer Nanocomposites, Editor J. K. Nelson, Eds. Springer, Heidelberg, Germany, 2010

[17] J. Claude, Y. Lu, Q. Wang, "Effect of molecular weight on the dielectric breakdown strength of ferroelectric poly(vinylidene fluoride chlorotrifluoroethylene)s, Applied Physics Letters, Vol. 91, pp. 2129041-212904-3, 2007

[18] K. Stark, C. Garton, "Electric strength of irradiated polythene", Nature, Vol. 176, pp. 1225-1226, 1955

[19] M. Nagao, T. Kimura, Y. Mizuno, M. Kosaki, M. Ieda, "Detection of Joule heating before dielectric breakdown in polyethylene films", IEEE Transactions on Electrical Insulation, Vol. 25, No. 4, pp. 715-722, 1990

[20] G. Kickelbick, "Introduction to hybrid materials", in: Hybrid Materials, Synthesis, Characterization and Applications, Editor G. Kickelbick, Eds. Wiley VCH Verlag GmbH\& Co. KGaA, Weinheim, Germany, 2007

[21] L. A. Dissado, J. C. Fothergill, "Electrical Degradation and Breakdown in Polymers", Eds. Peter Peregrinus Ltd., London, UK, 1992

[22] M. Roy, J. K. Nelson, R. K. MacCrone, L. S. Schadler, C. W. Reed, R. Keefe, W. Zenger, "Polymer nanocomposite dielectrics - The role of the interface", IEEE Transactions on Dielectrics and Electrical Insulation, Vol. 12, No. 4, pp. 629-643, 2005

[23] N. Fuse, M. Kozako, T. Tanaka, S. Murase, Y. Ohki, "Possible mechanism of superior partial discharge resistance of polyamide nanocomposites", Proceedings of Annual Report Conference on Electrical Insulation and Dielectric Phenomena, pp. 322-325, 2004

[24] T. Desai, P. Keblinski, S. Kumar, "Molecular dynamics simulations of polymer transport in nanocomposites", The Journal of Chemical Physics, Vol. 122, 134910-1-134910-8, 2005

[25] H. Lu, S. Nutt, "Restricted relaxation in polymer nanocomposites near the glass transition", Macromolecules, Vol. 36, pp. 4010-4016, 2003

[26] T. D. Fornes, D. R. Paul, "Crystallization behavior of nylon 6 nanocomposites", Polymer, Vol. 44, No. 14, pp. 3945-3961, 2003

[27] T. J. Lewis, "A model for nano-composite dielectrics under electrical stress", Proceedings of 2007 IEEE International Conference on Solid Dielectrics, pp. 11 -14, 2007

[28] G. Tsagaropoulos, A. Eisenberg, "Dynamic mechanical study of the factors affecting the two glass transition behavior of filled polymers. Similarities and differences with random ionomers. Macromolecules, Vol. 28, No. 18, pp. 6067-6077, 1995

[29] J. K. Nelson, Y. Hu, "The impact of nanocomposite formulations on electrical voltage endurance", Proceedings of International Conference on Solid Dielectrics (ICSD), pp. 832-835, 2004

[30] Y. Yamano, "Roles of polycyclic compounds in increasing breakdown strength of LDPE film", IEEE Transactions on Dielectrics and Electrical Insulation, pp. 774-781, 2006

[31] R. C. Smith, C. Liang, M. Landry, J. K. Nelson, L. S.Schadler, "The mechanisms leading to the useful electrical properties of polymer nanodielectrics", IEEE Transactions on Dielectrics and Electrical Insulation, Vol. 15, No. 1, pp. 187-196, 2008 
[32] M. Roy, J. K. Nelson, R. K. MacCrone, L. S. Schadler, "Candidate mechanisms controlling the electrical characteristics of silica/XLPE nanodielectrics", Journal of Materials Science, Vol. 42, 3789-3799, 2007

[33] M. Nagao, S. Watanabe, Y. Murakami, Y. Murata, "Water tree retardation of $\mathrm{MgO} / \mathrm{LDPE}$ and $\mathrm{MgO} / \mathrm{XLPE}$ nanocomposites", Proceedings of International Symposium on Electrical Insulating Materials (ISEIM 2008), pp. 483-486, Mie, Japan, September 7-11, 2008

[34] X. Dou, X. Liu, Y. Zhang, H. Feng, J.-F. Chen, S. Du, "Improved dielectric strength of barium titanate-polyvinylidene fluoride nanocomposite", Applied Physics Letters, Vol. 95, 132904-1-132904-3, 2009

[35] K. Yu, Y. Niu, F. Xiang, Y. Zhou, Y. Bai, H. Wang, "Enhanced electric strength and high energy density of barium titanite filled polymer nanocomposites", Journal of Applied Physics, Vol. 114, 174107-1174107-5, 2013

[36] T. P. Schuman, S. Siddabattuni, O. Cox, F. Dogan, "Improved dielectric breakdown strength of covalently bonded interface polymer-particle nanocomposites", Composite Interfaces, Vol. 17, No. 8, pp. 719-731, 2010

[37] S. Siddabattuni, T. P. Schuman, F. Dogan, "Improved polymer nanocomposite dielectric breakdown performance through barium titanate to epoxy interface control", Material Science \& Engineering B, Vol. 176, No. 18, pp. 1422-1429, 2011

[38] M. G. Danikas, R. Sarathi, "Interfaces in high voltage engineering: A most important question for conventional solid insulating materials as well as for nanocomposite polymers", Funktechnikplus\#Journal, Vol. 1, No. 4, pp. 7-31, 2014

[39] M. G. Danikas, A. Bairaktari, R. Sarathi, A. B. B. Abd. Ghani, "A review of two nanocomposite insulating materials models: Lewis' contribution in the development of the models, their differences, their similarities and future challenges", Engineering, Technololgy \& Applied Science Research, Vol. 4, No. 3, pp. 636-643, 2014

[40] L. Pietronero, H. Wiesmann, "Stochastic model for dielectric breakdown", Journal of Statistical Physics, Vol. 36, nos. 5/6, pp. 909916, 1984

[41] L. Niemeyer, L. Pietronero, H. J. Wiesmann, "Fractal dimension of dielectric breakdown", Physical Review Letters, Vol. 52, No. 12, pp. 1033-1036,1984

[42] H. J. Wiesmann, H. R. Zeller, "A fractal model of dielectric breakdown and prebreakdown in solid dielectrics", Journal of Applied Physics, Vol. 60, No. 5, pp. 1770-1773, 1986

[43] S. J. Dodd, "A deterministic model for the growth of non-conducting electrical tree structures", Journal of Physics D: Applied Physics, Vol. 36, No. 2, pp. 129-141, 2003

[44] M. G. Danikas, I. Karafyllidis, A. Thanailakis, A. M. Bruning, "Simulation of electrical tree growth in solid dielectrics containing voids of arbitrary shape", Modelling and Simulation in Materials Science and Engineering, Vol. 4, No. 6, pp. 535-552, 1996

[45] G. E. Vardakis, M. G. Danikas, I. Karafyllidis, "Simulation of spacecharge effects in electrical tree propagation using cellular automata", Materials Letters, Vol. 56, No. 4, pp. 404-409, 2002

[46] L. A. Dissado, P. J. J. Sweeney, "Physical model for breakdown structures in solid dielectrics", Physical Review B., Vol. 48, No. 22, pp. 16261-16268, 1993

[47] M. D. Noskov, A. S. Malinovski, M. Sack, A. J. Schwab, "Selfconsistent modeling of electrical tree propagation and PD activity", IEEE Transactions on Dielectrics and Electrical Insulation, Vol. 7, No. 6, pp. 725-733, 2000

[48] D. Pitsa. "Electrical Breakdown Phenomena and their modeling in nanocomposites", Doctoral Thesis, Department of Electrical and Computer Engineering, School of Engineering, Democritus University of Thrace , 2013 (in Greek)

[49] B. J. Cox, N. Thamwattana, J. M. Hill, "Electric field-induced force between two identical uncharged spheres", Applied Physics Letters, Vol. 88, pp. 152903-1-152903-3, 2006

[50] G. Chen, C. Zhang, G. Stevens, "Space charge in LLDPE loaded with nanoparticles", Proceedings of Annual Report Conference on Electrical Insulation and Dielectric Phenomena (CEIDP), pp. 275-278, 2007
[51] A. Hajiyiannis, G. Chen, C. Zhang, G. Stevens, "Space charge formation in epoxy resin including various nanofillers", Proceedings of Annual Report Conference on Electrical Insulation and Dielectric Phenomena (CEIDP), pp. 714- 717, 2008

[52] T. Iizuka, T. Tanaka, "Effects of nano silica filler size on treeing breakdown lifetimeof epoxy nanocomposites", Proceedings of International Conference on Properties and Applications of Dielectric Materials (ICPADM), pp. 733-736, 2009 